\title{
A Correlation Based Sensor Fusion Method for Multi-Channel Seismic Network
}

\author{
Mussab Zubair, Klaus Hartmann, and Otmar Loffeld, Senior Member, IEEE
}

\begin{abstract}
In this paper, an improved method of sensor-leve fusion for a multi-channel seismic network is presented for target detection in outdoor security applications. This method determines the weighted values of each sensor present in the seismic network based on the correlation measures and as the change of correlation is observed, the weighted values are adjusted accordingly for real-time data. This method takes full advantage of the limited range of seismic sensors especially in the outdoor environments since the sensors that reflect the state of target have greater weighted values than other sensors in a network. Furthermore, this method is also helpful to identify a failure state or bad data of the sensors. The effectiveness of this method is verified by simulation results. In the end, the application of this method is presented and the fusion results are discussed on real data.
\end{abstract}

Index Terms-Sensor fusion, correlation, multi-channel seismic network, target Detection.

\section{INTRODUCTION}

The target detection capability in a multi-channel seismic network is a challenging problem for outdoor security applications. The target produces seismic waves in the ground which are recorded by seismic sensors in a network. Although seismic waves are complicated to analyze as they propagate in different forms, in different directions, and at different propagation velocities and they are highly dependent on the underlying geology, these waves are less influenced by outdoor conditions like wind, Doppler effects and unwanted noises from the objects. Therefore, seismic sensors are suitable for outdoor applications.

In general, there are two categories of seismic waves: the body waves and the surface waves. Body waves travel at a higher speeds than surface waves and they propagate through the interior of the earth. Whereas, surface waves propagate near the surface of the earth. Research related to seismic waves indicates that a significant amount of seismic energy is dispersed near the surface if any kind of disturbance occurs near the surface of the earth and this energy is transmitted as the surface wave [1]. $70 \%$ of the total energy of the target is distributed as such Rayleigh surface waves and the remaining energy is propagated into the interior of the earth as body waves [2]. Thus, Rayleigh waves have more importance to

Manuscript received April 14, 2012; revised May 30, 2012.

M. Zubair gratefully acknowledges the support of DAAD on International Postgraduate Programme (IPP) MultiSensorics of the Center of SensorSystems (ZESS) at University of Siegen, Germany.

M. Zubair, K. Hartmann and O. Loffeld are with the Center of SensorSystems (ZESS), University of Siegen, Siegen 57076, Germany (e-mail: zubair@ ipp.zess.uni-siegen.de, Hartmann, Loffeld@ zess.uni-siegen.de). detect a target that makes impacts on the surface of the earth.

Although seismic sensors are appropriate for outdoor applications, they have the drawback of limited range [3], since the amplitude of Rayleigh wave is inversely proportional to the square root of the distance between the target and the seismic sensor [4]. To overcome this drawback, a technique of sensor network is applied where many seismic sensors are deployed in different locations to extract the information available in the environment. Many researchers have already worked on the problem of target detection in a multi-channel seismic network. The most common approach which is available in the scientific literature is to extract features of the target from each seismic sensor present in the network and fuse the information at the feature level to detect the target [5], [6]. This approach is usually computationally expensive because the number of processing steps and the size of the feature vectors increases proportionally with a number of sensors in the network. Apart from that, this approach does not incorporate any failure state of the sensors and as a result, the detection process performs poorly since bad data from these sensors contribute also in the detection process.

This paper presents a novel approach of sensor fusion to effectively deal with the problem of target detection in a multi-channel seismic network. In this approach, the preprocessed raw data from multi-channel seismic sensors are fused based on the correlation measures. Each sensor is correlated itself and all its neighbours in a network. The method calculates the weighted values of each sensor present in a network and their values are adjusted as changes of the correlation measures between neighbouring sensors are observed. In this way, this method takes full advantage of the limited range of seismic sensors in an outdoor environment. Since, as stated earlier, the amplitude of the seismic signal decreases with increasing distance between the target and the sensor, so the correlation between the sensor and its neighbouring sensors continuously changes. Furthermore, this method does not require previous values of sensor data to calculate the weighted values. So, this method yields higher measuring precision of the seismic signal and higher dynamic adaptability of real-time data. On the other hand, this method is also helpful to detect a failure state of sensor or to identify those sensors containing bad data. The sensors that contain no information about the target have low weighted values. Another advantage of using this method is that it provides flexibility to deal with sensors' data. It means that the features of the target can be extracted either from fused sensor data or from individual sensor data that reflect the presence of the target. In other words, the higher weighted values of sensors contain the information about the target. 
This paper is arranged as follows: section II presents some related work. Section III describes the detail description of the sensor fusion method. The simulation analysis is presented in section IV. The application and the results on real data are discussed in section V. And, finally, the conclusions are made in section VI.

\section{RELATED WORK}

In [7], a method of data fusion is presented on the basis of correlation function for the application of rotary machine fault diagnosis. The method performs cross-correlation operation between sensors to fuse signals. The method is not suitable to apply for target detection in multi-channel seismic network due to the following reasons. Firstly, that method cannot be applied to two sensors because the weighted values are always divided equally whether sensors contain the information of interest or not. Secondly, if the signals from sensors are uncorrelated, that method does not work.

In this paper, the above mentioned method is modified to overcome the drawbacks and to make the method suitable for outdoor security applications. Simulation results are presented to prove the effectiveness of the improved method. The results of both methods are compared and discussed on real data.

\section{SENSOR FUSION}

The weighted fusion model is represented by the following equation.

$$
X=w_{1} x_{1}+w_{2} x_{2}+w_{3} x_{3}
$$

where $X$ is the fused signal obtained from sensors, $x_{1}, x_{2}, \ldots, x_{n}$ are the signals of individual sensors, $w_{1}, w_{2}, \ldots ., w_{n}$ are the weighting coefficients of the individual sensor signals.

The weights are computed by performing auto-correlation operation on each sensor and cross-correlation operation between sensors in a network. These values are updated automatically for every new frame data.

The auto-correlation function quantifies the similarity between two segments of the same signal and is used to correlate the sensor data with itself. It gives the mean square value of sensor and is given by the following expression:

$$
R_{x x}(m)=\frac{1}{N-|m|} \sum_{n=1}^{N-m+1} x(n) x(n+m-1)
$$

where $(m=0,1,2, \ldots, k)$ and $k$ is known as time shift.

The cross-correlation function quantifies the similarity between two signals and is used to correlate one sensor signal with other sensors present in the network. It is used to find the correlation energy between sensors and is given by the following expression:

$$
R_{x y}(m)=\frac{1}{N-|m|} \sum_{n=1}^{N-m+1} y(n) x(n+m-1)
$$

where $(m=0,1,2, \ldots, k)$ and $k$ is known as time shift. He energy of the signal observed in each sensor can be expressed by the correlation measures. Therefore, the signal that has more energy will have greater correlation measure [8]. The total correlation energy of sensor $\mathrm{i}$ is determined by the following equation.

$$
E_{i}=\sum_{j=1}^{n}\left[R_{i j}\right]^{2}
$$

where $E_{i}$ is the total correlation energy of the signal observed in sensor $\mathrm{i}$ and $\mathrm{n}$ is the number of seismic sensors in a network.

As the weight of each sensor is directly proportional to the total correlation energy of the signal observed, we can write:

And

$$
w_{1}: w_{2}: \ldots . w_{n}=E_{1}: E_{2}: \ldots . E_{n}
$$

$$
w_{1}+w_{2}+\ldots+w_{n}=1
$$

From equation (4), (5) and (6), we can calculate the weighted values of each seismic sensor and using equation (1), we get the fused signal.

\section{A. Characteristics}

The weighted values of sensors determined from the method mentioned in section III have important characteristics.

- When there are no targets present in the network, sensors are considered to be uncorrelated and their weighted values are approximately equal, i.e.

$$
w_{1} \cong w_{2} \cong \ldots . \cong w_{n}
$$

- The presence of the target is indicated by higher weighting values of sensor.

- Sensors which contain no information about the target or contain bad data have lower weights. In this way, this method can detect failure of the sensor and that sensor can be discarded from fusion process.

- Since the weighted values are updated according to the change of correlation measures, the method shows good dynamic adaptability for real-time data.

- This method does not require previous values to compute the weighted values of each sensor.

- This method is simple and easy to use.

\section{Simulation Results AND Discussion}

In this section, simulation results of the improved method are presented and compared with the method presented in [7]. Two cases are considered for simulation. For all cases, the signal of interest is standard sine signal with a frequency of $1 \mathrm{~Hz}$.

\section{A. Case 1}

In the first case, it is assumed that two simulated sensors collect the vibration signals. Sensor 1 records a sine wave and sensor 2 captures white noise as shown in Figures $1 \mathrm{a}$ and $1 \mathrm{~b}$ respectively. The signals are normalized first with respect to the desired mean and variance, since the signals are not recorded with the same energy. The weighted values calculated from the improved method are as follows: 


$$
\begin{aligned}
& w_{1}=0.9924 \\
& w_{2}=0.0076
\end{aligned}
$$

While the method described in [7] cannot be applied to the two sensors since it always divides the weighted value equally for two sensors i.e., 0.5 independently of whether the signals contain useful information or not. This is one of the drawbacks which are overcome by this improved method.

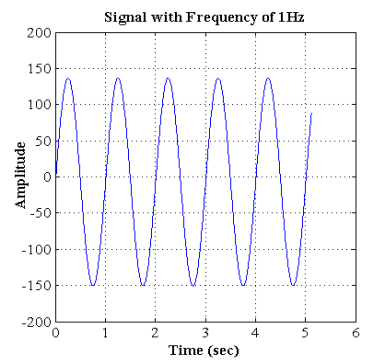

a)

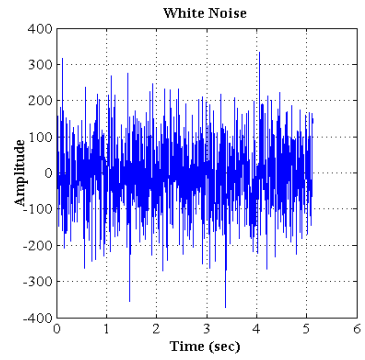

b)

Fig. 1. Vibration signal of two simulated sensors

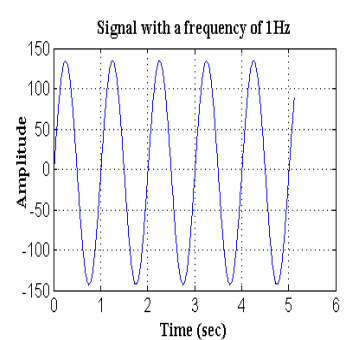

White Noise

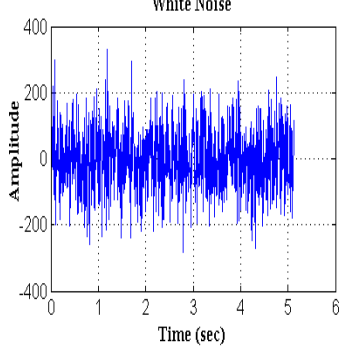

Fig. 2. vibration signal of three simulated sensors

\section{B. Case 2}

In this case, all three simulated sensors are considered to be uncorrelated. The sensor 1 records a sine signal, which is the signal of interest while sensor 2 and sensor 3 have white noises as shown in Figures 2a, 2b and 2c. The weighted values are calculated as follows:

$$
\begin{aligned}
& w_{1}=0.9777 \\
& w_{2}=0.0090 \\
& w_{3}=0.0133
\end{aligned}
$$

Fig. 2d shows the fused results of three signals. Again method explained in [7] cannot deal the problem of uncorrelated signals. For uncorrelated signals, the weighted values are always equal for all sensors. In this case, the weighted values of three simulating sensors are equal i.e., 0.3333 .

\section{ApPLICATION}

The improved method is effectively applied to an outdoor security application for personnel detection. The setup consists of five-channel seismic sensors in an area of 10 square meters as shown in Figure 3. Seismic sensors are deployed at different locations. The Z-axis signals of each sensor are considered for processing. The target "S" is moved along the specified path. For this application, the assumption is made that only a single person should be present in a test field at one time. The sensors are sampled at the rate of 51.2 $\mathrm{KHz}$. After preprocessing, the sampling rate is reduced to $512 \mathrm{~Hz}$. Since sensors are deployed in non-homogenous ground, it is possible that sensors may have different background energy levels. To compensate for this, all sensors in a network are normalized with respect to desired mean and variance before applying the sensor fusion method.

The weighted values of each sensor are calculated for every one second of real data. Since the person footsteps generate very weak signals, sensors that are close to the person record these signals. In this way, sensors that have higher weighted values represent the presence of the person in a network.

Fig. 4 and 5 provide the results of fusion based on the improved method and the method mentioned in [7]. Only sensors that have higher weighted values are fused using equation (1). It shows that the novel method provides higher energy of the footsteps as compared to the other method. Since the method explained in [7] is based on the cross-correlation function between the sensors, the higher energy levels are observed where sensors are highly correlated as shown in Fig. 5. The improved method also considers the mean square value of each sensor signal along with the correlation between sensors. From the fused signal in fig. 4, it is clear that sensor 5 has higher mean square value of the signal as compared to other sensors in a network
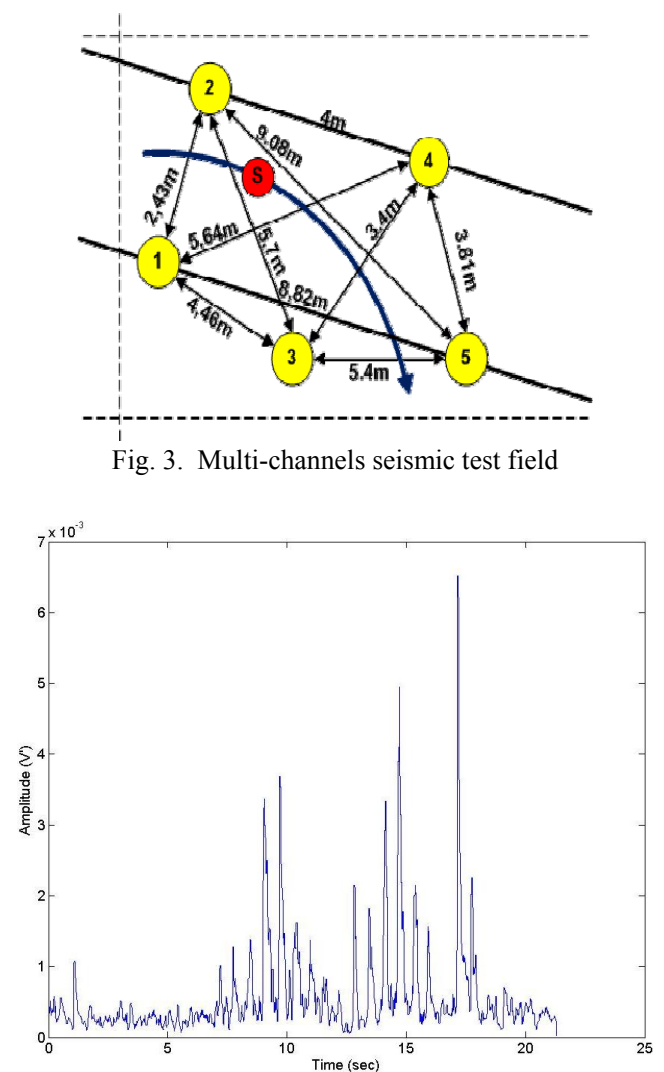

Fig. 4. Fused signal of person footsteps using novel approach 


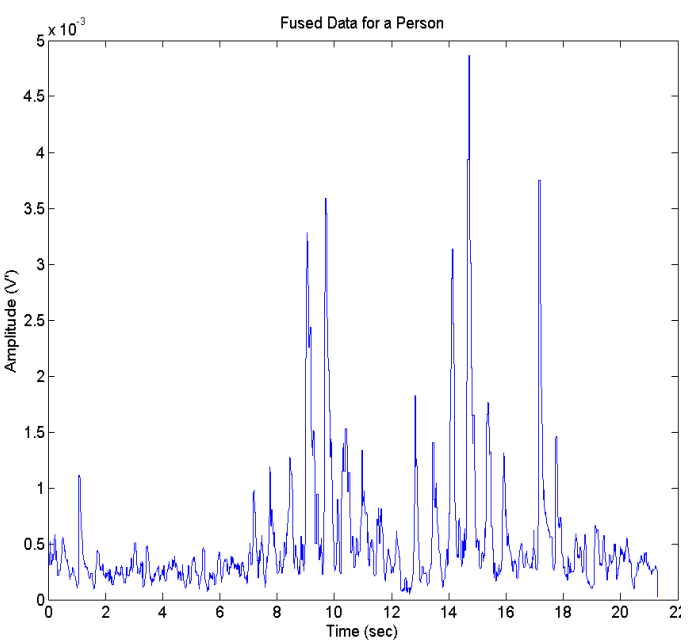

Fig. 5. Fused signal of person footsteps using method [7]

\section{CONCLUSION}

In this paper, an improved method of sensor level fusion for multi-channel seismic sensors has been presented. The method is especially effective for target detection in outdoor security applications. This method takes the advantage of the limited range of seismic sensors in the outdoor environment. The method does not need any data history or previous values for fusion. Since the calculation of the weighted values of these sensors are based on the correlation change of the real-time data, therefore, this method has good dynamic adaptability and fusion precision as compared to the traditional weighted average method. By the simulation results, we have shown that this method is also capable of handling uncorrelated signals. This method is also helpful to identify the sensor failure states and to reduce the computational burden by rejecting the sensor data which do not contain information about the target. The method also provides flexibility to deal with sensor data. Finally, it is concluded that the improved method is more sensitive to detect objects in a seismic network.

\section{ACKNOWLEDGMENT}

This work was supported and financed by the BSI (Federal Office for Information Security)-Project ZF-project 666c "POmSe". We would like to express our gratitude to all the colleagues who contributed to this project.

\section{REFERENCES}

[1] Y. Tian and H. Qi, "Target Detection and Classification using Seismic Signal Processing in Unattended Ground Sensor Systems," IEEE Conference on Acoustic, Speech and Signal Processing, vol. 4, 2002.

[2] G. Succi, G. Prada, R. Gampert, T. Pedersen, and H. Dhaliwal, "Problems in seismic detection and tracking," in Unattended Ground Sensor Technologies and Applications II. SPIE, vol. 4040, pp. 165-173, 2000.

[3] A. Pakhomov, A. Sicignano, M. Sandy, and T. Goldburt, "Current Seismic Sensor Issues for Defense and Security Applications," in Proc. of SPIE, vol. 5403, 2004

[4] V. D. Pavlovic and Z. S. Velickovic, "Measurement of the seismic waves propagation velocity in the real medium," Scientific journal FACTA UNIVERSITATIS, Series: Physics, Chemistry and Technology, vol. 1, pp. 63-73, 1998.

[5] H. Xing, F. Li, H. Xiao, Y. Wang, and Y. Liu, "Ground Target Detection, Classification and Sensor Fusion in distributed Fiber Seismic Sensor Network," in Proc. of SPIE, vol. 6830, pp. 1-10, 2008.

[6] D. Li, K. D. Wong, Y. H. Hu, and A. M. Sayeed, "Detection, Classification and Tracking of Targets in Distributed Sensor Networks," IEEE Signal Processing Magzine, vol. 19, pp. 17-29, 2002.

[7] L. Xuejun, B. Guangfu and B. S. Dhillon, "A new method of multi-sensor vibration signals data fusion based on correlation function," IEEE conference on Computer Science and Information Engineering, pp. 170-174, 2009

[8] L. Gupta, B. Chung, M. D. Srinath, "Multi-Channel fusion models for the parametric classification of differential brain acitivity," IEEE Transactions on Biomedical Engineering, pp: 1869-1882, 2005. 